\title{
Superconducting Pinning by Magnetic Domains in a Ferromagnet-Superconductor Bilayer
}

\author{
M.Z. Cieplak ${ }^{a}$, Z. Adamus ${ }^{a}$, M. KonczyKowski $^{b}$, \\ X.M. $\mathrm{CHEN}^{c}, \mathrm{~A} \cdot \mathrm{ByCZUK}^{a}, \mathrm{~A} \cdot \mathrm{ABAL}^{\prime} \mathrm{OSHEV}^{a}, \mathrm{HAI} \mathrm{SANG}^{c}$ \\ AND C.L. CHIEN ${ }^{c}$ \\ ${ }^{a}$ Institute of Physics, Polish Academy of Sciences \\ al. Lotników 32/46, 02-668 Warsaw, Poland \\ ${ }^{b}$ Laboratoire des Solides Irradies, CEA/DSM/DRECAM and CNRS-UMR 7642 \\ Ecole Polytechnique, 91128 Palaiseau Cedex, France \\ ${ }^{c}$ Department of Physics and Astronomy, The John Hopkins University \\ Baltimore, Md, 21218, USA

\begin{abstract}
The local flux profile and the critical current are studied using an array of Hall sensors in a ferromagnetic-superconducting bilayer which consists of niobium film covering ferromagnetic $\mathrm{Co} / \mathrm{Pt}$ multilayer with perpendicular magnetic anisotropy. The results indicate about threefold enhancement of the flux pinning in niobium layer caused by the isolated magnetic domains which are created during the magnetization reversal of the $\mathrm{Co} / \mathrm{Pt}$ multilayer. The geometrical barrier is absent, and the critical current is strongly peaked in close vicinity to the sample center, suggesting that the critical state differs from that predicted by the Bean model.
\end{abstract}

PACS numbers: 74.25.Ha, 74.25.Qt, 74.78.Db, 74.78.Fk

\section{Introduction}

The practical application of the superconducting (SC) films requires a high critical current density, which, in turn, depends on the pinning properties of the SC layers. Recently, it has been proposed theoretically that the flux pinning may be enhanced in a ferromagnet-superconductor $(\mathrm{FM} / \mathrm{SC})$ multilayer by magnetic domains which exist in the FM layer with perpendicular magnetic anisotropy [1]. 
Several experimental studies confirmed that this type of pinning enhancement occurs, both in multilayers and in the FM/SC bilayers (FSBs) [2-6]. In particular, we have studied the FSBs consisting of $\mathrm{Nb}$ film deposited on the top of $\mathrm{Co} / \mathrm{Pt}$ multilayer, and we found that the pinning is enhanced exclusively in the final stages of the magnetic reversal process of the FM layer. The effect is caused by the isolated bubble domains, which remain uninverted in the magnetic reversal process [6].

These studies probed either the transport critical current, or the global magnetization using the SQUID magnetometer. However, in a thin SC strip the critical current, $J_{\mathrm{c}}$, may be strongly nonuniform, depending both on the distance from the sample edge, $x$, and on the external magnetic field, $H$. To evaluate the dependence $J_{\mathrm{c}}(x, H)$ it is useful to measure the real flux distribution using the local magnetic field probe. In this work we measure the flux distribution in the FSB using the miniature Hall sensors placed on the top of the SC layer, while the pinning is adjusted by the magnetization of the FM layer.

\section{Experiment}

The FSBs were sputtered at room temperature on Si substrate as described in Ref. [6]. The structure, shown in Fig. 1a, consisted of $10 \mathrm{~nm}$ buffer layer of Pt, followed by 5 repeats of $\mathrm{Co}(0.4 \mathrm{~nm}) / \mathrm{Pt}(1 \mathrm{~nm})$ multilayer, $3 \mathrm{~nm}$ buffer layer of amorphous $\mathrm{Si}$ (to avoid the proximity effect), and $78 \mathrm{~nm}$ of $\mathrm{Nb}$ capped with a $3 \mathrm{~nm}$ of Si for protection. Niobium film had a superconducting transition temperature $T_{\mathrm{c}}=8.8 \mathrm{~K}$, the penetration depth $\lambda(0) \approx 95 \mathrm{~nm}$, and the coherence length $\xi(0) \approx 35 \mathrm{~nm}[6]$. Perpendicular magnetic anisotropy of the Co/Pt multilayer is evidenced by the rectangular shape of the hysteresis loop measured in SQUID magnetometer at $T=10 \mathrm{~K}$ (Fig. 1b). We describe the magnetic reversal process by the parameter $s$, which is equal to $1(0)$ when all the magnetic moments of the FM layer are "up" ("down") and the magnetization at saturation is equal $M_{\mathrm{s}}\left(-M_{\mathrm{s}}\right)$. At any other state with the magnetization $M, s=\frac{1}{2}\left(\frac{M}{M_{\mathrm{s}}}+1\right)$. We introduce also subscripts to $s$ to distinguish processes of reversal, that is $s^{+}\left(s^{-}\right)$ process is the one starting from $s=1(s=0)$. Figure 1c shows the magnetic force microscopy (MFM) image taken in the final stage of the magnetic reversal process, $s^{+}=0.2$. The dendrite-shaped, uninverted domains, about $0.5 \mu \mathrm{m}$ wide, are visible. The domain structure remains stable after the external field is turned off. As described in Ref. [6] these domains provide the largest enhancement of pinning in these FSBs.

The samples for the Hall sensor measurements were cut into $200 \mu \mathrm{m}$ wide strips from a larger piece of film by first drawing a line on $\mathrm{Si}$ substrate with a diamond scrapper, and breaking sample along that line. An array of eleven GaAs/AlGaAs Hall sensors, situated $20 \mu \mathrm{m}$ apart, was positioned across the SC strip (see the inset to Fig. 2). 
(a)

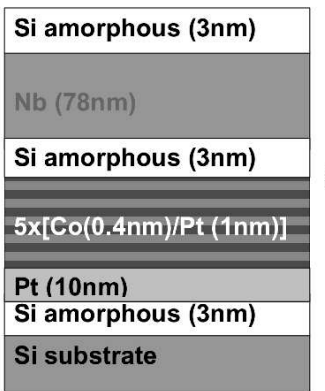

(b)

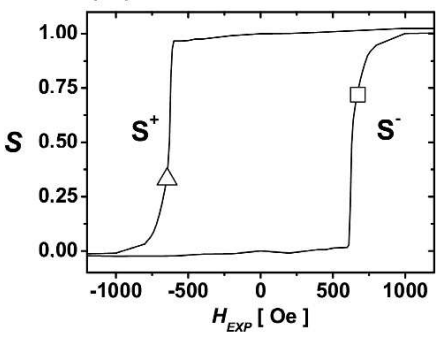

(c)

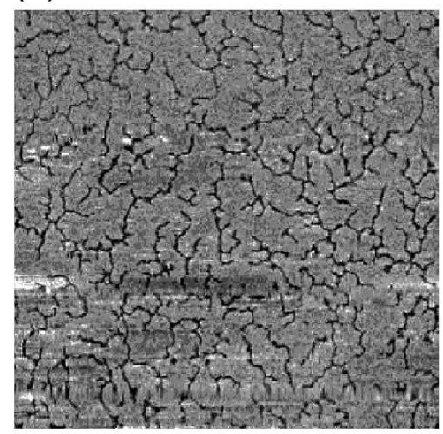

Fig. 1. (a) Schematic cross-section of the FSB structure. (b) Hysteresis loop of the Co/Pt multilayer at $T=10 \mathrm{~K}$. Open points show two magnetization states at which data in Fig. 3 are taken, $s^{-}=0.72$ and $s^{+}=0.33$. (c) MFM image taken at room temperature for $s^{+}=0.2$. The image size is $100 \times 100 \mu \mathrm{m}$.

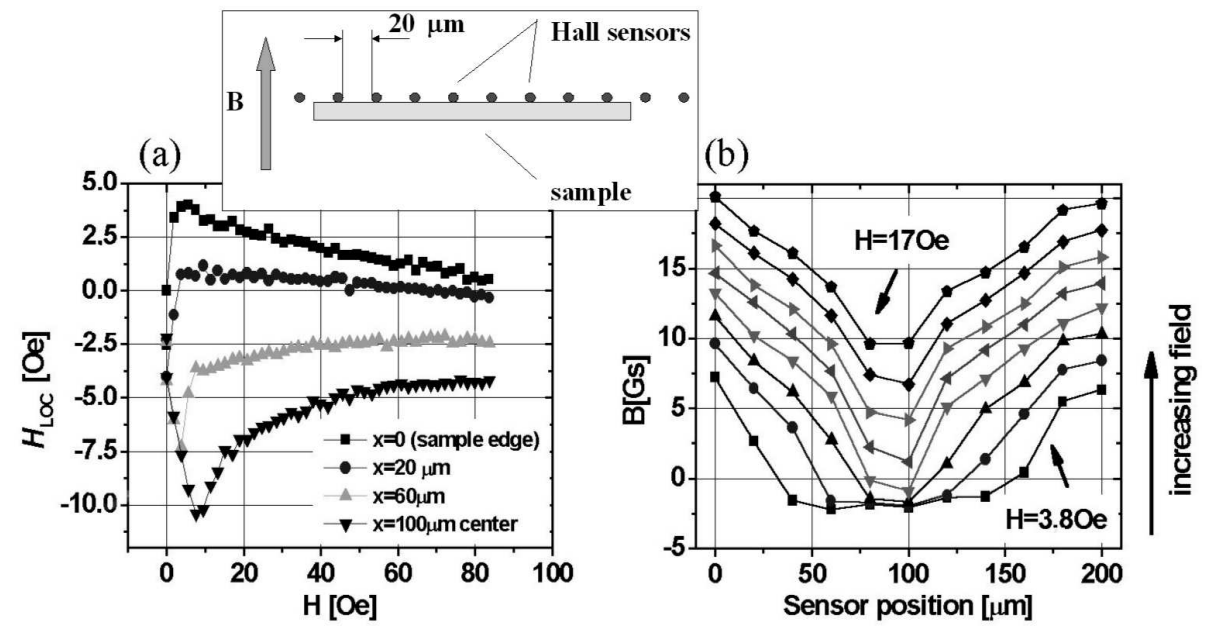

Fig. 2. The flux distribution for the intrinsic pinning $\left(s^{+}>0.93\right)$, taken at $T=7 \mathrm{~K}$ : (a) $H_{\mathrm{LOC}}(H)$ versus $H$ for several Hall sensors situated at a distance $x$ from the sample edge. (b) $B=H_{\mathrm{LOC}}+H$ as a function of $x$ for several values of $H$. The inset shows the arrangement of Hall sensors on the sample.

The measurements were performed as follows. First, at $T>T_{\mathrm{c}}$ large negative (or positive) magnetic field was applied to magnetize the FM layer up to $s^{-}=0\left(s^{+}=1\right)$. Next, the magnetic field was reversed and increased up to the magnitude $H_{\mathrm{EXP}}\left(-H_{\mathrm{EXP}}\right)$ to obtain some other value of $s^{-}\left(s^{+}\right)$. Finally, the field was turned off and the sample was cooled below $T_{\mathrm{c}}$. The local magnetic field above the SC layer, $H_{\mathrm{LOC}}$, was measured using all sensors simultaneously as the external magnetic field, $H$, was cycled between -80 Oe and 80 Oe. 


\section{Results}

We first examine the flux distribution when the FM layer is magnetized up to $s^{+}>0.93$, i.e. the magnetic domain pinning is absent and the flux pinning is intrinsic (Fig. 2). Part (a) shows the dependence of $H_{\mathrm{LOC}}(H)$ when $H$ is increased from zero value (zero-field cooled state), for several Hall sensors situated at a distance $x$ from the sample edge. Part (b) shows the magnetic induction, $B=$ $H_{\mathrm{LOC}}+H$, as a function of $x$ for several values of $H$. Both figures indicate that the magnetic field starts to penetrate the sample from the edge. This gives evidence of the absence of the geometrical barrier, which is sometimes detected in type II superconductors [7]. The profiles $B(x)$ shown in (b) for higher fields are not exactly linear. The linear profiles are predicted by the Bean model of the critical state in the SC slab with $J_{\mathrm{c}}=$ const. [8]. In the thin film geometry the deviations from linearity may occur [9]. Alternatively, the deviations may indicate that $J_{\mathrm{c}}$ is not constant, and should be more accurately described by the universal Kim model [10].

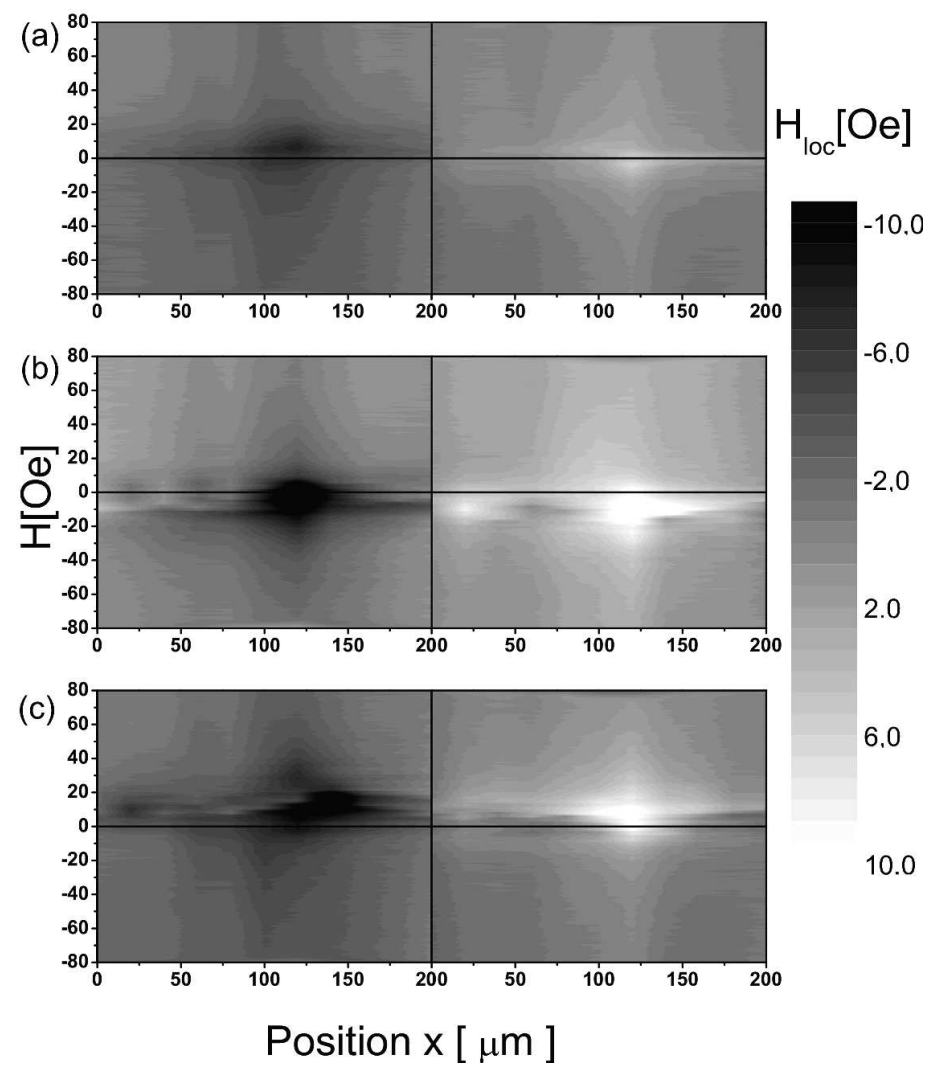

Fig. 3. Maps of $H_{\mathrm{LOC}}(H, x)$ measured at $T=7 \mathrm{~K}$ for three $s$-values: (a) intrinsic pinning, $s^{+}>0.9$, (b) $s^{-}=0.72$, and (c) $s^{+}=0.33$. 
Figure 3 shows maps of $H_{\mathrm{LOC}}(H, x)$ for three $s$-values. The graph (a) is for intrinsic pinning, $s^{+}>0.9$. The graphs (b) and (c) are for $s^{-}=0.72$ and $s^{+}=0.33$, respectively, when the FM layer is in the final stages of the magnetic reversal and contains a maze of dendrite-shaped domains as shown in Fig. 1c. Two effects are evident. First, the magnitude of $H_{\mathrm{LOC}}$ at the maximum is enhanced by almost 3 times in comparison with intrinsic pinning. Secondly, the maxima of $H_{\mathrm{LOC}}$, which are close to $H=0$ in (a), are shifted to positive (negative) $H$ for $s^{+}\left(s^{-}\right)$reversal process. This indicates that the enhancement of $H_{\mathrm{LOC}}$ is strongly asymmetric with respect to the relative orientation between the residual domains and the external magnetic field. This is expected since the domain dipole moment $m$ interacts with the vortex magnetic field $B$, and introduces a contribution to the vortex energy, $E=-m B$. Therefore, the positive domains $(m>0)$ which appear in the $s^{+}$reversal process, enhance the pinning for positive $H$ and negative domains $(m<0)$ in the $s^{-}$process produce the pinning for negative $H$.

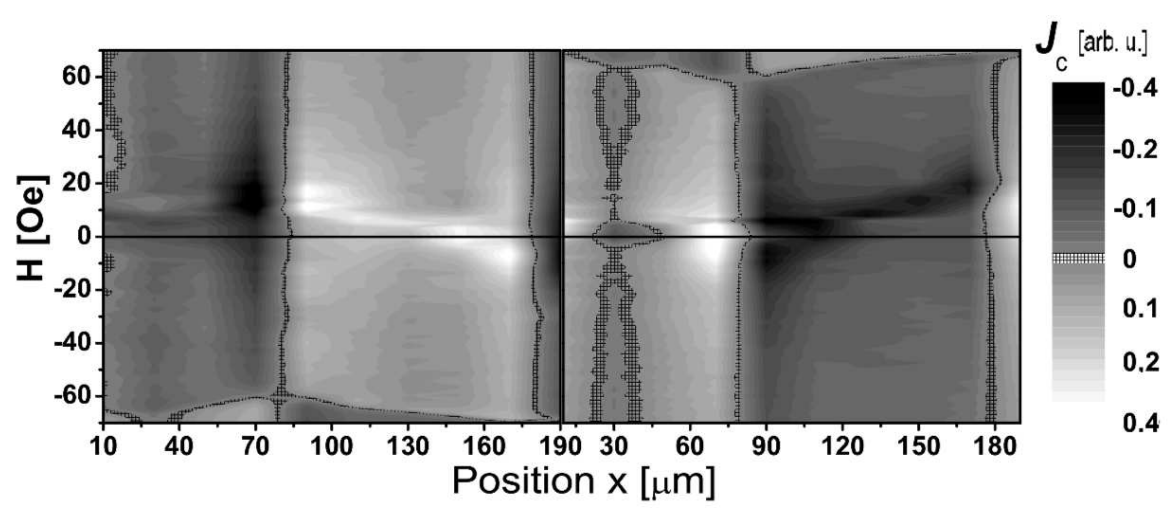

Fig. 4. The map of $J_{y}(H, x) \sim \mathrm{d} B / \mathrm{d} x . B(H, x)$ is measured at $T=7 \mathrm{~K}$ for $s^{+}=0.16$.

Finally, we evaluate the profile of the critical current, $J_{\mathrm{c}}(H, x)$, by calculating the gradient of the local magnetic induction along $x, J_{y}(x) \sim \mathrm{d} B / \mathrm{d} x$. Figure 4 shows one example of $J_{\mathrm{c}}(H, x)$ map, measured for $s^{+}=0.16$. $J_{\mathrm{c}}$ is very nonuniform across the sample, it has sharp maxima and minima in the close vicinity of the sample center where it changes the sign. This type of the profile is inconsistent with the Bean model of the critical state [8], and suggests that the universal Kim model is more appropriate [10].

\section{Conclusions}

The profiles of the local magnetic field and the critical current were measured in the FSB structure which consists of $\mathrm{Nb}$ layer on the top of $\mathrm{Co} / \mathrm{Pt}$ multilayer. The local magnetic field in the SC layer is enhanced by the isolated magnetic domains created in the FM layer by almost 3 times in comparison to the intrinsic pinning. 
The geometrical barrier is absent in these FSBs. It is found that the critical current is strongly peaked in a close vicinity to the sample center, suggesting that the critical state differs from that predicted by the Bean model.

\section{Acknowledgments}

This work was supported by the State Committee for Scientific Research (Poland) grant 2 P03B 044 23, and by the NSF grant No. DMR00-80031.

\section{References}

[1] L.N. Bulaevskii, E.M. Chudnovsky, M.P. Maley, Appl. Phys. Lett. 76, 2594 (2000).

[2] A. Garcia-Santiago, F. Sanchez, M. Varela, J. Tejada, Appl. Phys. Lett. 77, 2900 (2000).

[3] X.X. Zhang, G.H. Wen, R.K. Zheng, G.C. Xiong, G.J. Lian, Europhys. Lett. 56, 119 (2001).

[4] M. Lange, M.J. Van Bael, V.V. Moshchalkov, Y. Bruynseraede, Appl. Phys. Lett. $\mathbf{8 1}, 322(2002)$.

[5] D.B. Jan, J.Y. Coulter, M.E. Hawley, L.N. Bulaevskii, M.P. Maley, Q.X. Jia, B.B. Maranville, F. Hellman, X.Q. Pan, Appl. Phys. Lett. 82, 778 (2003).

[6] M.Z. Cieplak, X.M. Cheng, C.L. Chien, Hai Sang, unpublished.

[7] E. Zeldov, A.I. Larkin, V.B. Geshkenbein, M. Konczykowski, D. Majer, B. Khaykovich, V.M. Vinokur, H. Shtrikman, Phys. Rev. Lett. 73, 1428 (1994).

[8] C.P. Bean, Phys. Rev. Lett. 8, 250 (1962).

[9] E. Zeldov, J.R. Clem, M. McElfresh, M. Darwin, Phys. Rev. B 49, 9802 (1994).

[10] D.-X. Chen, R.B. Goldfarb, J. Appl. Phys. 66, 2489 (1989). 